\title{
Galaxy and Mass Assembly (GAMA): luminosity function evolution
}

\author{
Jon Loveday and the GAMA team \\ Astronomy Centre, University of Sussex, Falmer, Brighton BN1 9QH, UK \\ email: J.Loveday@sussex.ac.uk
}

\begin{abstract}
We describe modifications to the joint stepwise maximum likelihood method of Cole (2011) in order to simultaneously fit the GAMA-II galaxy luminosity function (LF), corrected for radial density variations, and its evolution with redshift. The whole sample is reasonably well-fit with luminosity $(Q)$ and density $(P)$ evolution parameters $Q, P \approx 0.8,1.7$. Red galaxies show larger luminosity but smaller density evolution than blue galaxies, as expected.
\end{abstract}

Keywords. galaxies: evolution, galaxies: luminosity function, mass function, galaxies: statistics

\section{Introduction}

The luminosity function (LF) is perhaps the most fundamental model-independent quantity that can be measured from a galaxy redshift survey. Reproducing the observed LF is the first requirement of a successful model of galaxy formation, and thus accurate measurements of the LF are important in constraining the physics of galaxy formation and evolution (e.g. Benson et al. 2003). In addition, accurate knowledge of the survey selection function (and hence LF) is required in order to determine the clustering of a flux-limited sample of galaxies (Cole 2011).

A standard $1 / V_{\max }$ (Schmidt 1968) estimate of the LF is sensitive to radial density variations within the sample. This sensitivity can be largely mitigated by multiplying the maximum volume $V_{\max }$ in which each galaxy is visible by the integrated radial overdensity of a density-defining population (Baldry et al. 2006, 2012). Maximum-likelihood methods (Sandage et al. 1979; Efstathiou et al. 1988) are also unaffected by density fluctuations due to galaxy clustering. However, if the sample covers a significant redshift range, galaxy properties (such as luminosity) and number density are subject to systematic evolution with lookback time. All of the above methods must then either be applied to restricted redshift subsets of the data, or be modified to explicitly allow for evolution (e.g. Lin et al. 1999; Loveday et al. 2012).

Cole (2011) recently introduced a joint stepwise maximum likelihood (JSWML) method, which jointly fits non-parametric estimates of the LF and the galaxy overdensity in radial bins, along with an evolution model. In this paper we adapt Cole's JSWML method in order to determine the LF and its evolution from the Galaxy and Mass Assembly (GAMA) survey (Driver et al. 2011).

Throughout, we assume a Hubble constant of $H_{0}=100 \mathrm{~h} \mathrm{~km} / \mathrm{s} / \mathrm{Mpc}$ and an $\Omega_{M}=$ $0.3, \Omega_{\Lambda}=0.7$ cosmology in calculating distances, co-moving volumes and luminosities.

\section{Parametrizing the evolution}

We parametrize luminosity and density evolution using the parameters $Q$ and $P$ introduced by Lin et al. (1999). Evolution in absolute magnitude is assumed to be linear 
with redshift, $E(z)=Q\left(z-z_{0}\right)$, such that absolute magnitude $M$ is determined from apparent magnitude $m$ using

$$
M=m-5 \log _{10} d_{L}(z)-25-K\left(z ; z_{0}\right)+Q\left(z-z_{0}\right),
$$

where $d_{L}(z)$ is the luminosity distance at redshift $z$ and $K\left(z ; z_{0}\right)$ is the $K$-correction, relative to a passband blueshifted by $z_{0}$, determined from fitting a spectral energy distribution to the model ugriz magnitudes for each galaxy using KCORRECT V4_2 (Blanton et al. 2003; Blanton \& Roweis 2007). Luminosity evolution is determined relative to the same redshift $z_{0}$ as the $K$-correction. In order to minimize errors introduced by uncertainties in individual galaxy $K$-corrections and evolution histories, one should set $z_{0}$ close to the mean redshift of the sample. Here, we choose $z_{0}=0.1$ so that results may be directly compared with a previous estimate of the GAMA LF and its evolution (Loveday et al. 2012).

Evolution in number density $\bar{n}(z)$ is parametrized as

$$
\bar{n}(z)=\bar{n}\left(z_{0}\right) 10^{0.4 P\left(z-z_{0}\right)}=\bar{n}(z=0) 10^{0.4 P z} .
$$

\section{Maximum likelihood density-corrected $V_{\max }$ method}

We have adapted the Cole (2011) method by (i) including incompleteness-correction weights and (ii) finding optimum evolution parameters by minimising the combined $\chi^{2}$ from radial overdensities and the comparison of the evolution-corrected LF in redshift slices:

$$
\chi^{2}=\sum_{i} \frac{\left(\phi_{i}^{z_{1 \mathrm{o}}}-\phi_{i}^{z_{\mathrm{hi}}}\right)^{2}}{\operatorname{Var}\left(\phi_{i}^{z_{1 \mathrm{o}}}\right)+\operatorname{Var}\left(\phi_{i}^{z_{\mathrm{hi}}}\right)}+\sum_{p} \frac{\left(\Delta_{p}-1\right)^{2}}{\sigma_{p}^{2}},
$$

where $\phi$ is estimated in two broad redshift ranges $z_{\text {lo }}$ and $z_{\text {hi }}$ split near the mean redshift of the sample, $\bar{z} \approx 0.2 ; \operatorname{Var}(\phi)$ is the corresponding variance, determined by jackknife resampling; $\Delta_{p}$ is the overdensity in radial bin $p$ and $\sigma_{p}^{2}$ its variance, also determined by jackknife resampling.

We first evaluate $\chi^{2}$ values on a rectangular grid of $(P, Q)$, thus allowing one to visualise the correlations between the evolution parameters. The grid point with the smallest $\chi^{2}$ value is then used as a starting point for a downhill simplex minimisation to refine the parameter values corresponding to minimum $\chi^{2}$.

\section{Results}

Fig. 1 shows $\chi^{2}$ contours in the space of the evolution parameters $P, Q$ determined using equation (3.1) for the full GAMA-II sample and for blue and red galaxies separately. The multi-modal nature of the likelihood contours is most likely due to changes in the noise properties of individual LF bins as the varying value of $Q$ moves galaxies from one luminosity bin to another. $\dagger$ We see a significant trend of increasing density evolution $P$ from red to blue galaxies. This is to be expected, since many galaxies that were starforming and hence blue at higher redshift have since ceased forming stars and now lie on the red sequence, e.g. Peng et al. (2010). Less significantly, one also observes a trend of decreasing luminosity evolution $Q$ from red to blue, again as expected since the luminosity of the latter population is maintained by ongoing star formation. The differences between red and blue galaxies agree qualitatively with those of Loveday et al. (2012), although in

$\dagger$ We are currently investigating a method which fits a smooth kernel density estimate of the $\mathrm{LF}$, thus avoiding these binning effects. 


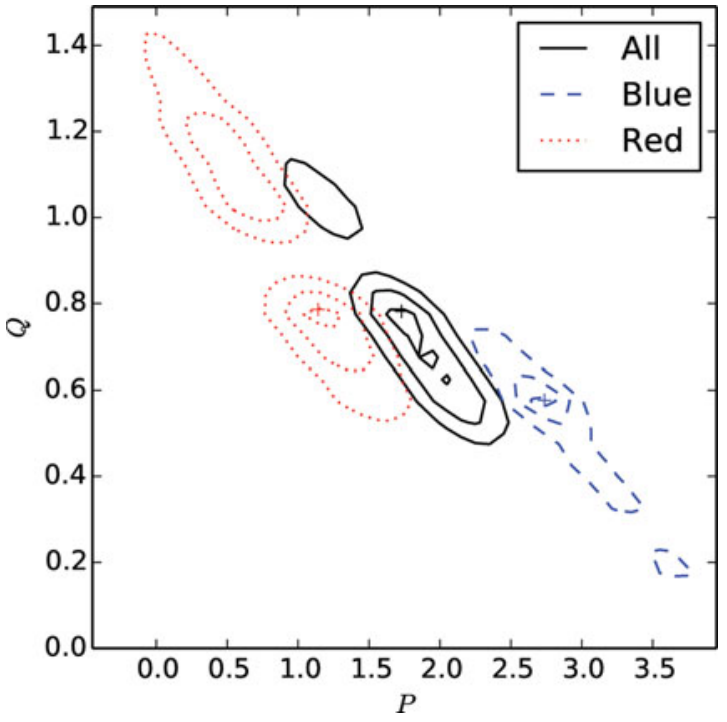

Figure 1. 1, 2, and $3 \sigma \chi^{2}$ contours for GAMA-II evolution parameters for all, blue and red galaxies as labelled. The plus signs indicate the location of minimum $\chi^{2}$.

Table 1. Best-fitting evolution and $r$-band LF parameters. $\chi_{\mathrm{ev}}^{2} / \nu$ is the reduced $\chi^{2}$ from equation (3.1), $\chi_{\phi}^{2} / \nu$ is the reduced $\chi^{2}$ from least-squares Schechter function fits to the LF estimates; none of the LFs are well-fit in detail by a Schechter function, particularly at the bright end. The uncertainties quoted on the LF parameters come from jackknife sampling, but do not explicitly include the large degeneracies between parameters.

\begin{tabular}{lccccccc}
\hline Sample & $Q$ & $P$ & $\chi_{\text {ev }}^{2} / \nu$ & $\alpha$ & $M^{*}-5 \log h$ & $\log \phi^{*} / h^{3} \mathrm{Mpc}^{-3}$ & $\chi_{\Phi}^{2} / \nu$ \\
\hline All & 0.78 & 1.73 & 3.18 & $-1.28 \pm 0.07$ & $-20.76 \pm 0.06$ & $-2.06 \pm 0.05$ & 2.11 \\
Blue & 0.58 & 2.74 & 3.77 & $-1.47 \pm 0.06$ & $-20.53 \pm 0.08$ & $-2.41 \pm 0.06$ & 1.54 \\
Red & 0.79 & 1.14 & 3.09 & $-0.71 \pm 0.14$ & $-20.57 \pm 0.07$ & $-2.15 \pm 0.05$ & 2.78 \\
\hline
\end{tabular}

the present analysis we no longer see any evidence for negative density evolution for red galaxies. See Table 1 for best-fitting evolution parameters.

Radial overdensities are shown in the left panel of Fig. 2; Petrosian $r$-band LFs are shown in the right panel. Surface brightness and redshift incompleteness have been taken into account by appropriately weighting each galaxy. We have fit a Schechter function to each binned LF using least squares, the parameters are tabulated in Table 1. Note that the Schechter fit for red galaxies grossly underestimates the faint end of the LF. It is likely that the faint-end upturn for red galaxies is at least partly due to the inclusion of dusty spirals; the stellar mass function of E-Sa galaxies of Kelvin et al. (in prep.) shows no indication of a low-mass upturn. In detail, none of the LFs are well-described by Schechter functions, with large reduced $\chi^{2}$ values. This is due to the high statistical precision of the GAMA data (jackknife errors for most LF bins are smaller than the plotting symbols), resulting in the large statistical significance of apparently small deviations from a Schechter function. There are significantly more high-luminosity $\left(M_{r}-5 \lg h<-23\right.$ mag) galaxies than predicted by the Schechter function fit. Since these very luminous galaxies lie at high redshift, this could be due to non-linearity in true luminosity evolution. These LFs are consistent with the $r$-band LFs determined from the GAMA-I sample by Loveday et al. (2012), using slightly different methods, and shown in the Figure as dotted lines. We also show the 'corrected' LF from the Blanton et al. (2005) low-redshift SDSS sample (without colour selection). Considering that we are comparing the LFs of SDSS galaxies within only $150 h^{-1} \mathrm{Mpc}$ with GAMA galaxies out to $z \approx 0.65$, the agreement 


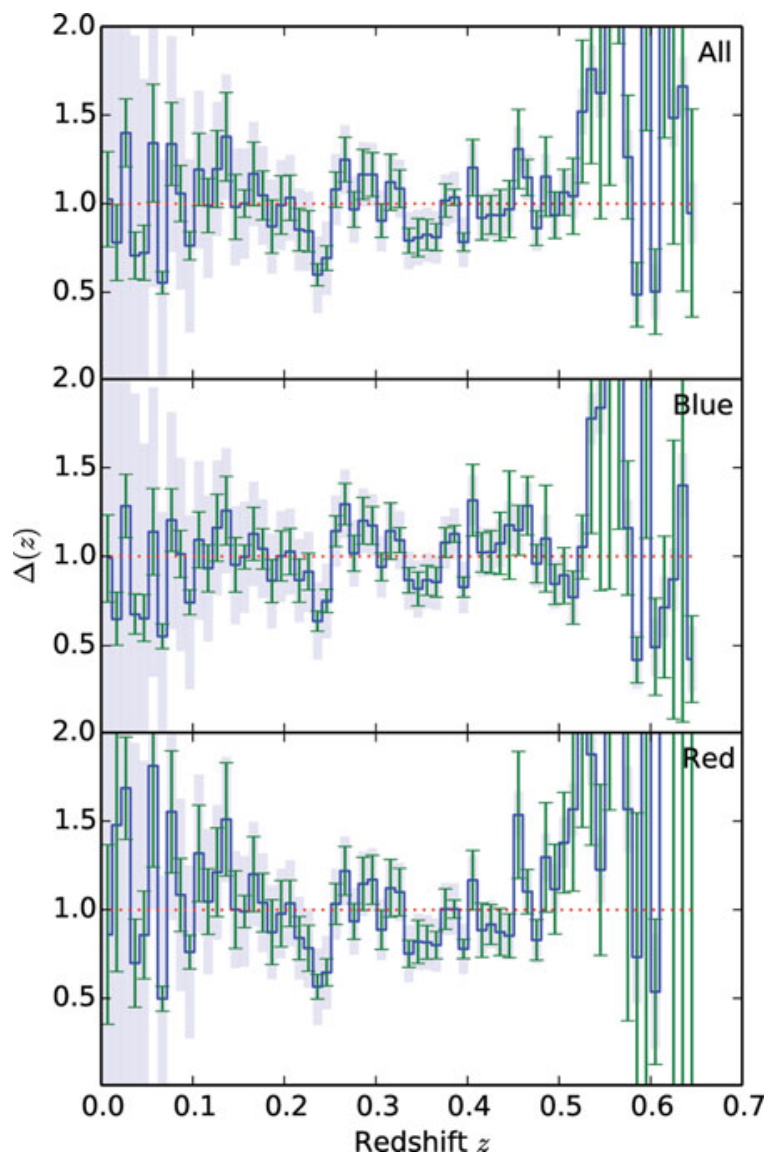

Figure 2. Left: radial overdensities determined from GAMA-II using the entire sample and blue and red subsets as labelled. The error bars show uncertainties estimated from jackknife sampling and the shaded regions show the expected variance assuming $J_{3}=\int r^{2} \xi(r) d r \approx 2,000 h^{-3} \mathrm{Mpc}^{3}$. Right: GAMA-II evolution- and density-corrected Petrosian $r$-band LFs with best-fitting Schechter functions (solid lines) assuming evolution parameters for each sample as given in Table 1. The dotted lines show the best-fit $r$-band Schechter functions from Table 5 of Loveday et al. (2012). The open diamonds in the top panel show the 'corrected' LF from Fig. 7 of Blanton et al. (2005).

is remarkably good, and provides further evidence that our simple evolutionary model allows one to accurately recover the evolution-corrected LF.

The GAMA website is: http://www.gama-survey.org/.

\section{References}

Baldry I. K., Balogh M. L., Bower R. G., Glazebrook K., Nichol R. C., Bamford S. P., Budavari T., 2006, MNRAS, 373, 469

Baldry I. K. et al., 2012, MNRAS, 421, 621

Benson A., Bower R., Frenk C., Lacey C., Baugh C., Cole S., 2003, ApJ, 599, 38

Blanton M. R. et al., 2003, AJ, 125, 2348

Blanton M. R., Lupton R. H., Schlegel D. J., Strauss M. A., Brinkmann J., Fukugita M., Loveday J., 2005, ApJ, 631, 208

Blanton M. R., Roweis S., 2007, AJ, 133, 734

Cole S., 2011, MNRAS, 416, 739 
Driver S. P. et al., 2011, MNRAS, 413, 971

Efstathiou G., Ellis R. S., Peterson B. A., 1988, MNRAS, 232, 431

Lin H., Yee H. K. C., Carlberg R. G., Morris S. L., Sawicki M., Patton D. R., Wirth G., Shepherd C. W., 1999, ApJ, 518, 533

Loveday J. et al., 2012, MNRAS, 420, 1239

Peng Y.-j. et al., 2010, ApJ, 721, 193

Sandage A., Tammann G. A., Yahil A., 1979, ApJ, 232, 352

Schmidt M., 1968, ApJ, 151, 393

Taylor E. N. et al., 2011, MNRAS, 418, 1587 